\title{
Research on Vehicle Lane-Change Driving Behavior Based on Optimal Velocity Model
}

\author{
Ma Yu-yue, Wang Ji-zhong, Gao Li, Zhang Hui \\ School of Mechanical and Automotive Engineering, Qingdao University of Technology, Qingdao, China \\ Email address: \\ 534276015@qq.com (Ma Yu-yue), wangjizhong@qtech.edu.cn(Wang Ji-zhong), 740316864@qq.com (Gao Li), \\ 635559726@qq.com (Zhang Hui)
}

\section{To cite this article:}

Ma Yu-yue, Wang Ji-zhong, Gao Li, Zhang Hui. Research on Vehicle Lane-Change Driving Behavior Based on Optimal Velocity Model. International Journal on Data Science and Technology. Vol. 4, No. 2, 2018, pp. 60-66. doi: 10.11648/j.ijdst.20180402.14

Received: May 17, 2018; Accepted: June 1, 2018; Published: July 4, 2018

\begin{abstract}
Vehicle lane-change driving behavior affects the safety of vehicle driving and the stability of traffic flow, and it has great significance to establish a reasonable lane-change driving behavior model for studying lane-change driving characteristics and developing driver assistance system. The influence of the associated vehicle driving state on the lane-change behavior during the changing process is analyzed, and the driving behavior model based on optimal velocity model is established by using the vehicle following theory. The Theil's $U$ objective function is used to calibrate the model parameters, the prediction results of the model are compared with the actual measured results. The study shows that the lane-change behavior can be approximately described as the two kinds of car following behavior in the original lane and the target lane to the front car. The lane-change model established can truly describe the lane-change driving characteristics.
\end{abstract}

Keywords: Optimal Velocity Model, Lane-Change Driving Behavior, Lane-Change Model, Parameter Calibration

\section{Introduction}

Vehicle lane-change driving is a common behavior in multi-lane traffic flow. It has a direct impact on the safety of vehicle driving and the stability of traffic operation. Studying vehicle lane-change driving behavior and building reasonable modeling method are of great significance for the design and development of the driver's auxiliary system and the vehicle automatic driving system to ensure the safety of the road traffic.

Scholars at home and abroad have studied vehicle driving behavior from different angles. For example, Gipps [1] established a decision structure model of urban road change early, which took into account the influence of traffic signals, obstacles and vehicle types on the changing behavior; Zhang Y. et al [2] established the MRS changing model and concluded that the changing motive was mainly determined by the characteristics of the driver and the stimulus from the external driving environments; Kesting et al. [3] proposed a lane changing model to judge vehicle lane changing behavior and avoid risk by using longitudinal acceleration; Zheng Z. [4] put forward the vehicle changing rule through studying the traffic simulation model; Talebpour [5] proposed a vehicle routing model based on game theory and verified it by experimental data; and Shi [6], combining the vehicle following process with the changing process, established the longitudinal acceleration model in the process of the vehicle arbitrariness based on the full velocity difference model. Wang [7] put forward a car following model with two front cars based on two models of the full velocity difference following and the probability lane changing. There have more researched on the lane changing rules [8,9], while study on the comprehensive influence of the driving state of the associated vehicle and the lateral displacement of the changing vehicle on the change of the driving behavior is so less that the lane changing characteristics under the typical lane-change case cannot be well reflected.

In this paper, vehicle lane-change driving behavior is analyzed, and the classic vehicle-following model that only considering the influence of single car information on the following car is extended to the a new, which considering the influence of driving states on original lane and target lane on the changing vehicle. A model of driving behavior based on the optimal velocity following model is established, also a reasonable model of parameter calibration is put forward to 
verify the correctness of the lane-changing model.

\section{Analysis of Lane Changing Behavior}

\subsection{Vehicle Changing Behavior}

According to the different driving motivation, the behavior of vehicle changing can be divided into mandatory and random characters [10], no matter what the changing behavior should be realized through the driver's perception decision - execution. The driver should make a series of decisions in a short time [11, 12]. Figure 1 shows a typical sketch of the vehicle lane change.

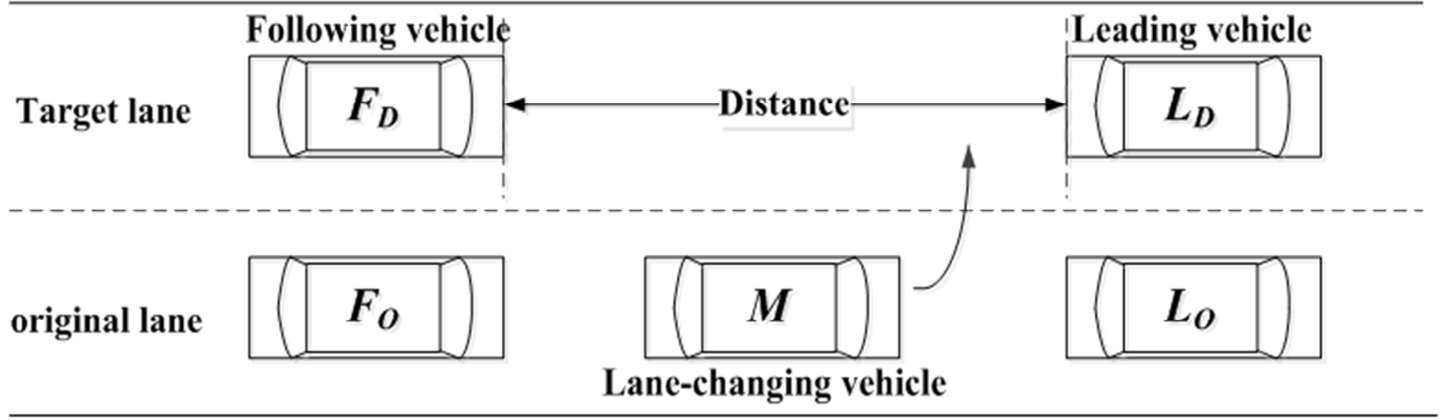

Figure 1. Sketch of vehicle typical lane-change scene.

The driver of the lane-change vehicle $M$, perceiving the driving state of the vehicle and the surrounding vehicles according to the intention, make a decision on changing the lane and adjust the state of the vehicle for changing, also pay close attention to the driving state of the vehicle in the original lane and the target lane in order to timely adjust lane changing decisions and lane changing behavior under the changes in surrounding conditions. In the changing process, the driving states of vehicle $L_{O}$ as leading car in the original lane and vehicle $L_{D}$ as leading car in the target lane have the most obvious influence on the vehicle $M$ lane-changing driving. Therefore, the influence of the leading vehicle on the lane-changing vehicle only is considered in this study, without regard for the information of other vehicles.

During lane changing, the behavior of lane-change vehicle $M$ from starting the changing intention to completing the lane changing behavior smoothly can be divided into two stages. In the first stage, the vehicle $M$ is following the leading vehicle in the original lane. At the same time, the changing vehicle is approaching to the target lane and looking for the right chance to cross the lane line. In the other stage, the lane-changing vehicle is crossing the lane and driving on the target lane, which can be regarded as a vehicle-following behavior of the leading vehicle on the target lane.

\subsection{Change of the Weight Coefficient in Lane-Changing}

The longitudinal and lateral displacements of the lane-change vehicle are produced simultaneously during the lane-changing process. Due to the lateral displacement, the driving state of leading vehicle in original lane and target lane has different effects on lane-change vehicles, resulting in the different driving behavior. Therefore, when establishing lane-change models, it is necessary to consider comprehensively the influence of leading vehicles in different lanes on driving behavior of lane changing vehicle.

The weight coefficient $\kappa$ and $\lambda$ respectively express the degree of concern to the leading vehicle on the original lane and the target lane for lane-change vehicle $M$. The weight coefficient varies with the lateral displacement of the lane-change vehicle, which respectively reflects the influence degree of leading vehicles in each lane on lane-changing vehicle driving behavior during the changing. Figure 2 shows the change of attention degree of lane changing vehicles to relevant vehicles during the lane changing.

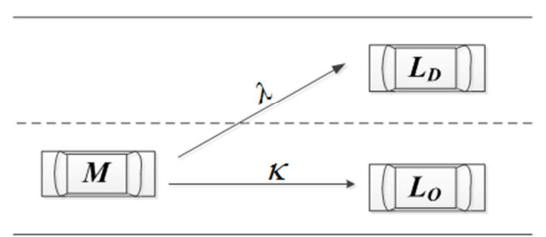

(a) Starting changing

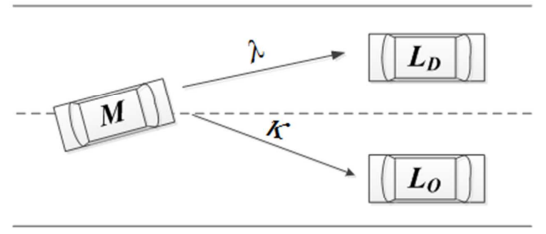

(b) Crossing the lane

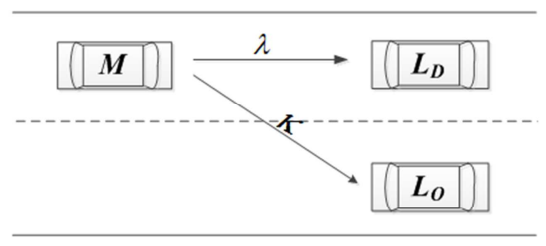

(c)Finishing changing

Figure 2. Schematic diagram of the attention change for lane-change vehicle to related vehicles during changing process.

As mentioned above, the lane-changing vehicle $M$ can be regarded as a kind of following behavior of leading vehicle when it is running normally in the original lane. The driving behavior is mainly influenced by the driving state of the leading vehicle, so the value of coefficient $\kappa$ is bigger in the first lane-change stage. In the course of changing the path, with the continuous increase of the lateral displacement, lane changing vehicle $M$ gradually left the original lane and 
approached the target lane. The lane-change vehicle $M$ is gradually reduced by the influence of leading vehicle $L_{O}$ on the original lane and more attention is paid to the change of the driving state of leading vehicle $L_{D}$ on target lane, so the value of coefficient $\lambda$ is gradually increased. When the lane-change vehicle $M$ completely crosses the lane line, it is no longer affected by the influence of vehicle on the original lane, and becomes a new vehicle-following behavior on the target lane.

\section{The Establishment of Vehicle Change Behavior Model}

\subsection{Optimal Velocity Following Model}

Vehicle following refers to the process of following the forward vehicle when the vehicle is lined up in a single lane without overtaking. The typical vehicle following process is shown in Figure 3. Vehicle following model is an important microscopic traffic flow model to study vehicle following behavior.

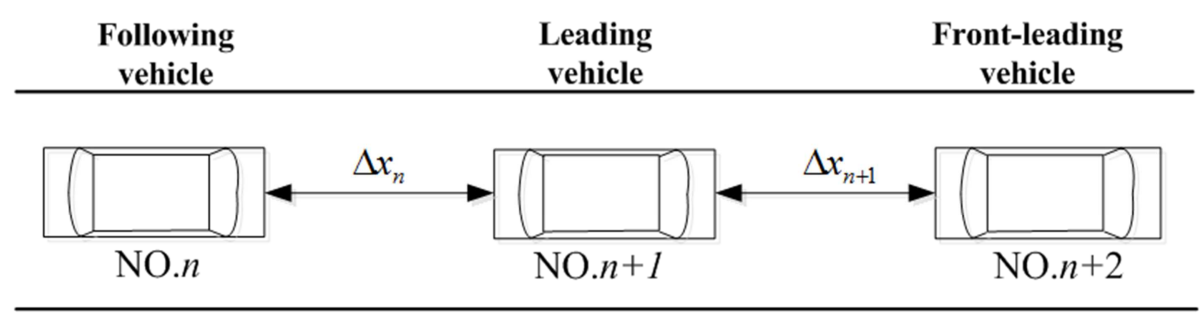

Figure 3. The diagrammatic sketch of vehicle following.

In 1995, Bando et al [13] put forward the Optimal Velocity model based on the speed optimization function of vehicle spacing. It is expressed as:

$$
\frac{d^{2} x_{n}}{d t^{2}}(t)=\alpha\left[V\left(\Delta x_{n}(t)\right)-v_{n}(t)\right]
$$

Where $\alpha$ represents the reaction sensitivity coefficient of drivers; $x_{n}(t)$ and $v_{n}(t)$ represent the position and speed of the vehicle $n$ at the $t$ moment; $\Delta x_{n}(t)=x_{n+1}(t)-x_{n}(t)$ represents the distance between the front vehicle and the rear car at the $t$ moment; $V\left(x_{n}(t)\right)$ represents the optimization speed function for vehicle spacing $\Delta x_{n}(t)$, that is:

$$
V\left(x_{n}(t)\right)=\frac{v_{\max }}{2}\left[\tanh \left(\Delta x_{n}(t)-h_{c}\right)+\tanh \left(h_{c}\right)\right]
$$

Where $v_{\max }$ represents the maximum vehicle speed; $h_{c}$ represents the safe distance between vehicles.

\subsection{The Establishment of a Lane-Changing Model}

During changing lane, the driving behavior of lane-changing vehicle is divided into three stages: perception-decision-execution. In the perception stage, lane changing drivers perceive the driving state of the current vehicle and the surrounding vehicles. The perception variables usually include the location, speed, acceleration of the vehicle, the location, speed and acceleration of the surrounding vehicles, as well as the distance between the vehicle and the surrounding vehicle, the relative speed, etc. In the decision stage, lane changing drivers make decisions on perceived variables according to different rules and driving behavior models, and the decision variables are generally the acceleration or speed of the vehicle. At the execution stage, the driver adjusts the driving state according to the driving decision [14]. Taking the acceleration of lane changing vehicle as the decision variable, the schematic diagram of the lane changing model is shown in Figure 4.

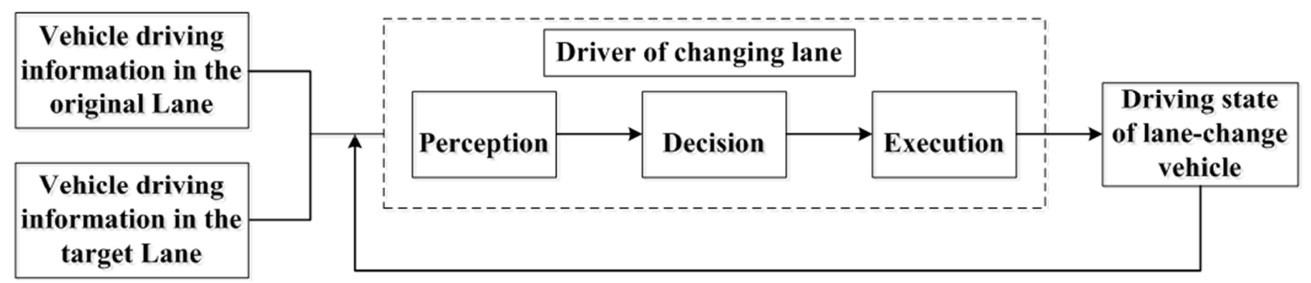

Figure 4. Diagram of driving behavior model.

The Optimal Velocity model can simulate many actual traffic flow phenomena, but the model only considers the influence of the distance between the following vehicle and the leading vehicle on the driving behaviors of the following vehicle on a single road. While in the changing process, through the above analysis, the lane-change driving behavior can be approximately described as the linear combination of two following behaviors of the leading vehicle $L_{O}$ on original lane and the leading vehicle $L_{D}$ on target lane, that is, the decision variables of the acceleration of the lane-change vehicle can be expressed as: 


$$
\frac{d^{2} x_{M}}{d t^{2}}(t)=\kappa \frac{d^{2} x_{M-L O}}{d t^{2}}(t)+\lambda \frac{d^{2} x_{M-L D}}{d t^{2}}(t)
$$

Where, $\frac{d^{2} x_{M-L O}}{d t^{2}}(t), \frac{d^{2} x_{M-L D}}{d t^{2}}(t)$ respectively the decision variables of lane-changing vehicle $\mathrm{M}$ that is affected by the leading vehicle separately in the original lane and target lane, and $\frac{d^{2} x_{M}}{d t^{2}}(t)$ respectively the decision variables of lane-changing vehicle $\mathrm{M}$ under the comprehensive influence.

\subsection{Changing Rules of Weight Coefficient}

In the process of changing the lane, taking the left lane-change as an example, the lateral position of the lane-change vehicle is restricted. The longitudinal direction of the vehicle is $x$ axis and the lateral migration is $y$ axis. The shadow part in Figure 5 shows the start and terminal range of the lane-changing vehicle. Where $\omega$ represents the width of the vehicle, $\mathrm{H}$ represents the width of the lane.

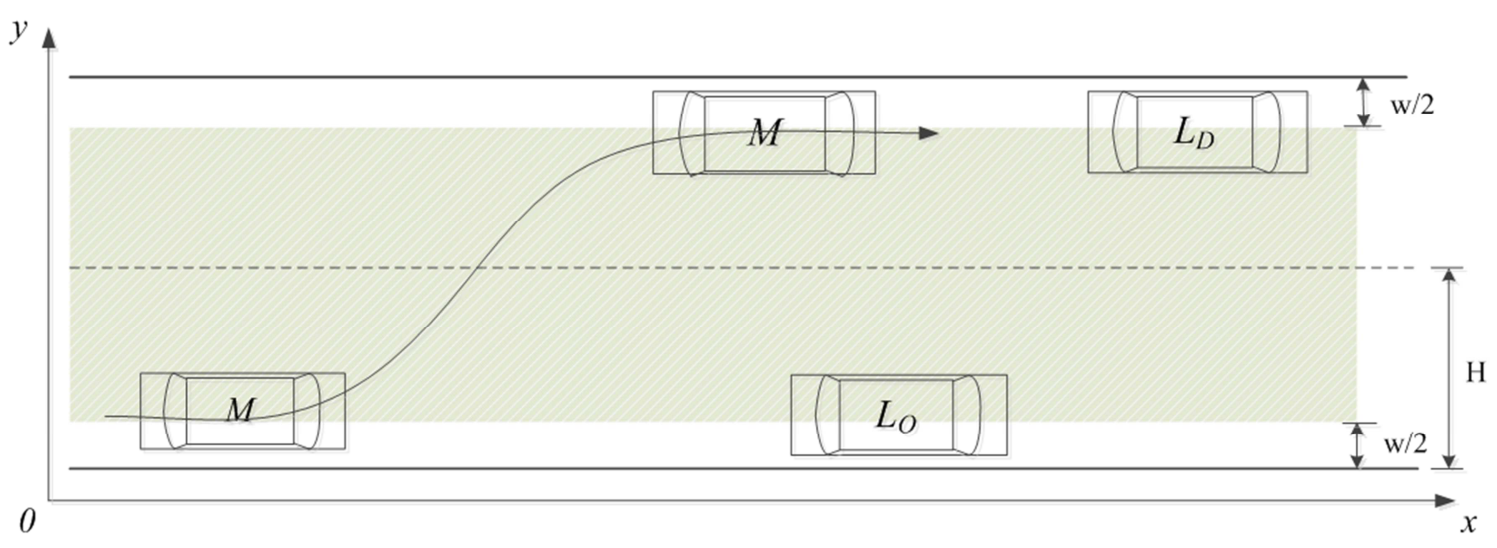

Figure 5. Position constraint for lane-change vehicle.

According to the change of lateral displacement and attention degree in the process of changing, the variation of concern coefficients about leading vehicles on the original lane and the target lane for the lane-change vehicle can be approximated linearly with the change of the lateral displacement. In the first stage of lane changing, the expression of coefficient $\kappa$ with lateral migration is
$\kappa=\left(1-\frac{y}{H}\right) \kappa_{0}$, and $\kappa_{0}$ is the initial value. The increase of $\lambda$ is the decrease of $\kappa$. At the second stage of lane changing, the lane-change vehicle is no longer affected by the influence of $\kappa$, only focusing on the influence of the leading vehicle on the target lane. The relationship between the weight values and the lateral migration of lane changing vehicle is shown in Figure 6.

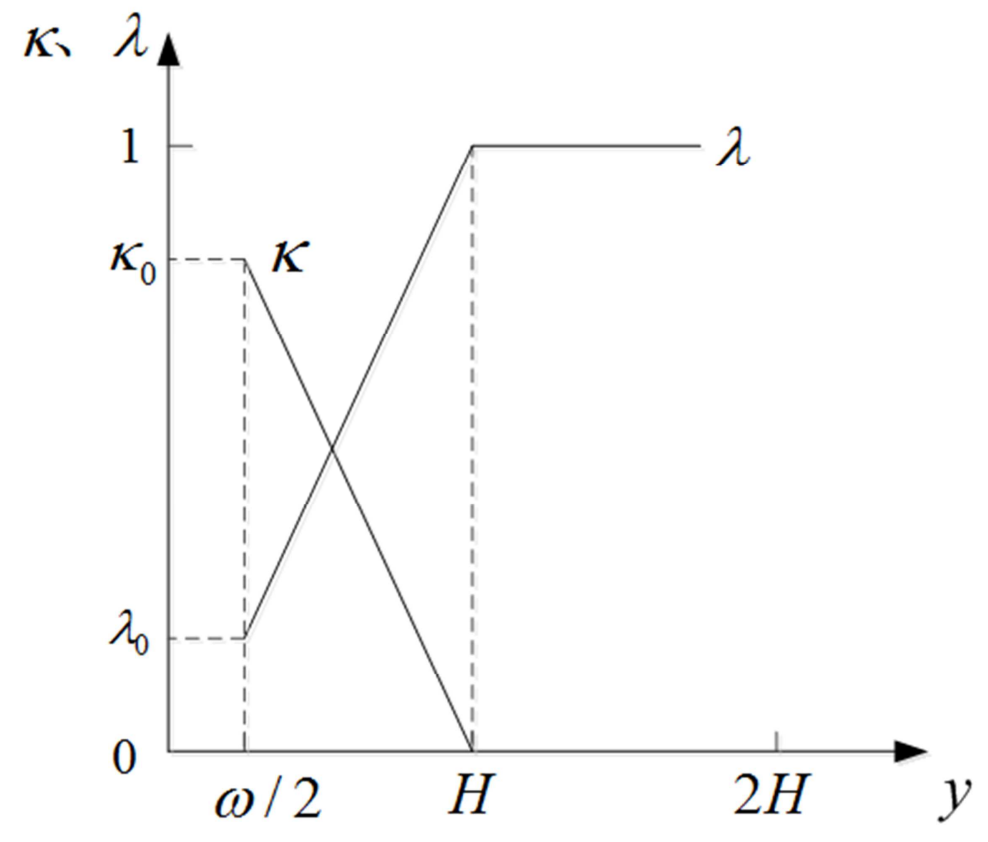

Figure 6. Variation of coefficient values with lateral migration. 
Put formula (1) and (2) into formula (3), the model of lane-change driving behavior based on the optimal velocity following model is established, then it is expressed as:

$$
\begin{aligned}
& \frac{d^{2} x_{M}}{d t^{2}}(t)=\kappa\left\{\alpha_{1}\left[0.5 V_{\max 1}\left[\tanh \left(x_{L O}(t)-x_{M}(t)-h_{c 1}\right)+\tanh \left(h_{c 1}\right)\right]-v_{M}(t)\right]\right\} \\
& +\lambda\left\{\alpha_{2}\left[0.5 V_{\max 2}\left[\tanh \left(x_{L D}(t)-x_{M}(t)-h_{c 2}\right)+\tanh \left(h_{c 2}\right)\right]-v_{M}(t)\right]\right\}
\end{aligned}
$$

The determined parameters of the lane-change driving behavior model are: $\kappa_{0} 、 \lambda_{0} 、 V_{\max 1}, V_{\max 2} 、 \alpha_{1} 、 \alpha_{2}, h_{c 1}$ 、 $h_{c 2}$.

\section{Parameter Calibration of Vehicle Lane-Change Driving Behavior Model}

The purpose of parameter calibration is to keep the traffic simulation data from the model to be consistent with the actual traffic data. By constructing the objective function, it is used to reflect the difference between the simulation data of the model and the real data, and then to find the parameters that make the objective function minimum, that is, the value of the calibrated parameter.

\subsection{The Determination of the Objective Function}

The constraint condition of objective function is the effective range of parameters in the model. We use the following general formula to express the parameter calibration problem in microscopic traffic model. Then, it is expressed as:

$$
\begin{gathered}
\min _{\Omega} f(\Omega, Y, \hat{Y}) \\
r_{i}(\Omega) \geq 0, i=1,2, \ldots, m
\end{gathered}
$$

Where, $f(\Omega, Y, \hat{Y})$ is an objective function, $\Omega$ represents the set of all parameters in the model. $Y 、 \hat{Y}$ are used to describe the vehicle running state, which can be the speed, acceleration, distance between the vehicles or the position of the vehicle. $Y$ represents the actual traffic data and $\hat{Y}$ represents the prediction data from simulation. $r_{i}(\Omega) \geq 0, i=1,2, \ldots, m$ represents the nonlinear constraint of model parameters, which is generally the effective range of parameters to be calibrated.

The acceleration of the lane-change vehicle as the decision variable in the model, the objective function of the model parameter calibration is established by using Theil's U function. It is:

$$
U=\frac{\sqrt{\frac{1}{N} \sum_{i=1}^{N}\left(\hat{a}_{i}-a_{i}\right)^{2}}}{\sqrt{\frac{1}{N} \sum_{i=1}^{N}\left(\hat{a}_{i}\right)^{2}}+\sqrt{\frac{1}{N} \sum_{i=1}^{N}\left(a_{i}\right)^{2}}}
$$

Where $i$ represents the experimental sample numbers, $N$ represents the sample capacity and $a_{i}$ is the acceleration observation value of lane-change vehicle NO. $i, \hat{a}_{i}$ is the corresponding model prediction result.

\subsection{Parameter Calibration of Lane-Changing Model}

The Binhai Road of Huangdao Development Zone in Qingdao is selected as the data collection point, and the driving states of 50 vehicles at different time are randomly recorded. The test data are processed according to the real-time monitoring video and running track of the vehicle to to determine the changing behaviors and the starting and stopping points of the vehicles. For vehicle $n, r_{\mathrm{n}}(t)$ represents the vehicle location at any time $t, \Delta t$ represents data sampling time interval, $r_{\mathrm{n}}\left(t_{\mathrm{i}}\right)$ represents the vehicle location at the time of data sampling $t_{\mathrm{i}}$.

$$
r_{n}\left(t_{i}+\Delta t\right) \neq r_{n}\left(t_{i}\right)
$$

If the upper form is satisfied, the vehicle $\mathrm{N}$ can be determined to occur the lane changing behavior, and the time of crossing the lane also can be determined.

The driving information of the lane changing vehicle $M$ and the associated vehicle $L_{O}$ and $L_{D}$ at the sampling time point is put into the lane-change model, the model output for parameter calibration can be obtained. The data features in the vehicle lane changing are shown in Table 1.

Table 1. Real data features in vehicle lane-changing.

\begin{tabular}{lll}
\hline The time for changing & The longest time & $8.7 \mathrm{~s}$ \\
lane & The shortest time & $3.4 \mathrm{~s}$ \\
& The average time & $4.93 \mathrm{~s}$ \\
The speed of & Maximum driving speed & $15.3 \mathrm{~m} / \mathrm{s}$ \\
lane-change vehicle & Minimum driving speed & $8.6 \mathrm{~m} / \mathrm{s}$ \\
& Average driving speed & $13.78 \mathrm{~m} / \mathrm{s}$ \\
& Maximum acceleration & $3.5 \mathrm{~m} / \mathrm{s}^{2}$ \\
The acceleration of & Maximum deceleration & $3.0 \mathrm{~m} / \mathrm{s}^{2}$ \\
lane-change vehicle & Average acceleration & $0.23 \mathrm{~m} / \mathrm{s}^{2}$ \\
& Mean Absolute Deviation of & $1.7 \mathrm{~m} / \mathrm{s}^{2}$ \\
\hline
\end{tabular}

50 groups of lane changing data were randomly divided into two groups: training and testing. The training data is used to calibrate the parameters of the model by solving the minimum value problem of the objective function, and the testing data is used to examine the calibration results of the model parameters. The minimum value of the objective function is solved by genetic algorithm [15]. The calibration results of model parameters are shown in Table 2. 
Table 2. Parameter calibration results of lane-change driving behavior model.

\begin{tabular}{|c|c|c|c|c|c|c|c|c|}
\hline parameter & $\kappa_{0}$ & $\lambda_{0}$ & $\alpha_{1}$ & $\alpha_{2}$ & $V_{\max 1}(\mathbf{m} / \mathbf{s})$ & $V_{\max 2}(\mathrm{~m} / \mathbf{s})$ & $h_{c 1} \quad$ (m) & $h_{c 2} \quad(\mathbf{m})$ \\
\hline Calibrated value & 0.72 & 0.28 & 0.59 & 0.41 & 13.23 & 16.56 & 8.6 & 12.07 \\
\hline
\end{tabular}

The calibration index of model parameters is expressed by the Mean Absolute Error of the predicted values and the measured values at each sampling point. Then, it is expressed as

$$
M A E=\frac{1}{N} \sum_{i=1}^{N}\left|\hat{a}_{i}-a_{i}\right|
$$

$M A D$ reflects the range of driver uncertainty in vehicle trajectory data to a certain extent. It is generally believed that if the MAE error of the calibration parameter is within the range of $M A D$ error, the parameter calibration results can reach the acceptable range [16]. The expression of $M A D$ is as follows:

$$
M A D=\frac{1}{N} \sum_{i=1}^{N}\left|a_{i}-\bar{a}\right|
$$

According to the calibration results and testing data of model parameters, the calculation of the calibrated evaluation index of model is that $M A E=1.65 \mathrm{~m} / \mathrm{s}^{2}$. It is known from table 1 that the calculation of the mean absolute deviation of the acceleration from the actual data is $1.70 \mathrm{~m} / \mathrm{s}^{2}$. It is shown that the calibrated error of the model parameters is smaller, and the calibration results of model parameters are effective.

According to the calibration result, the value of $\kappa_{0}$ is greater than that of $\lambda_{0}$ at the beginning stage of the lane changing. It shows that the driver of the lane-changing vehicle pays more attention to the driving state of the leading vehicle on the original lane rather than the vehicle on the target lane at the initial time of the changing lane, which is in accordance with the actual lane-change driving behavior, that is to say that the calibrated results are reasonable.

\subsection{Comparison of Model Prediction Results}

Taking the one lane-changing process of a vehicle in the experimental data as an example, comparing the actual data of changing lane with the calibrated data of model, the prediction value of the acceleration of lane-changing vehicle with time can be obtained. Figure 7 shows the acceleration predicted value of the model and the actual acceleration value along with time.

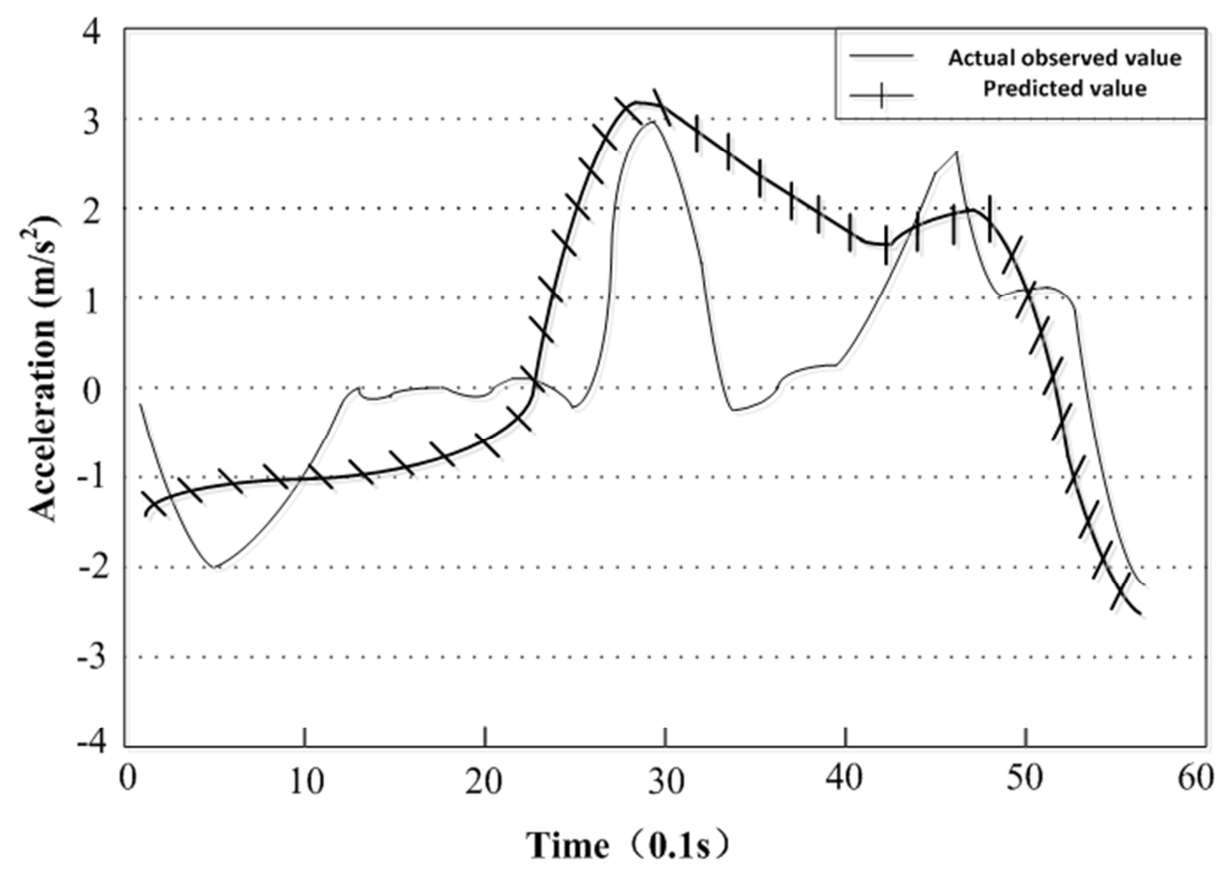

Figure 7. The predicted and observed values of lane-change vehicle acceleration with time.

It shows that the predicted value of the lane-change model based on the optimal velocity following model is consistent with the real value of the actual lane-change driving, and the correctness of the model is also proved. But at the same time, there are still great differences between the predicted value and the true value in some parts, which is due to the large difference between the driving behavior characteristics of different drivers, and the driving behavior is influenced by the driver's age, sex, driving age and education. The model prediction results can only be statistically as close to the overall characteristics of the sample population as possible, but cannot ensure that the predicted results of each sample are 
fully consistent with the actual values.

\section{Conclusion}

The behavior of lane-change vehicle in the changing process is affected by the driving state of the surrounding related vehicles and the lateral migration itself, based on the classic vehicle-following theory, which can be approximately divided into several combinations of the following behavior. The model of vehicle lane-change driving behavior by using the Their's $U$ target function and experimental data to effectively calibrate the parameters of the changing model, is proved that can accurately reflect the vehicle driving behavior characteristics. Hope provide reference for design and development of driver assistance system and vehicle automatic driving system to ensure road safety.

\section{References}

[1] Gipps P G. A model for the structure of lane-changing decisions [J]. Transportation Research Part B: Methodological, 1986, 20(5): 403-414.

[2] Zhang Y, Owen L. EA Multi-Regime Microscopic Traffic Simulation Approach [C] // Fifth International conference on Applications of Advanced Technologies in Transportation Engineering. 1998.

[3] Kesting A, Treiber M, Helbing D. General lane-changing model MOBIL for car-following models [J]. Transportation Research Record: Journal of the Transportation Research Board, 2007, 199(1): 86-94.

[4] Zheng Z. Recent developments and research needs in modeling lane changing $[\mathrm{J}]$. Transportation Research Part B; Methodological, 2014, 60(1): 16-32.

[5] Talebpour A., Mahmassani H. S., Hamdar S. H. Modeling lane-changing behavior in a connected environment: A game theory approach $[\mathrm{J}]$. Transportation Research Part C: Emerging Technologies, 2015, 7: 420-440.
[6] Shi, B. Y., Yang, X. G., Yu, X. F. Consider the free lane changing model combined with the car following model and simulate [J]. Traffic science and engineering, 2009, 25 (4): 91-96.

[7] Wang H., Liu Z., Zhang Z., et al. Considering the combined model of car following and lane changing for dual lead vehicles [J]. Journal of Southeast University Natural Science Edition, 2015, 45 (5): 985-989.

[8] Zheng Y. B. M. Study on road traffic behavior before Road intersection [D]. Hefei: HeFei University of Technology, 2017.

[9] Qu D. Y., Li J., Liu C. et al. Research on vehicle lane changing behavior based on hierarchical Logit model [J]. science and technology and engineering, 2017, 17 (5): 307-311.

[10] Huo, K. Driver lane changing behavior and gaze transfer characteristics of urban road [D]. Xi'an: Chang'an University, 2010 .

[11] Han, Z. Modeling of driver's vehicle Agent micro lane changing behavior [D]. University of Science \& Technology China, 2011.

[12] Sun, J., Li K. P., Yang, X. G. Simulation of lane changing behavior in crowded traffic flow weaving area [J]. System simulation journal, 2009, 21 (13): 4174-4182.

[13] Bando M, Hasebe K, Nakayama A, et al. Dynamical model of traffic congestion and numerical simulation [J]. Physical Review E, 1995, 51(2):1035-1042.

[14] Chang, H. Vehicle lane changing driving assistance method under vehicle road coordination [D]. Beijing: Beijing Jiaotong University, 2015.

[15] Wang, D. H., Tao, P. F., Jin, S., et al. Parameter calibration and verification method of car following model [J]. Journal of Jilin University: Engineering Edition, 2011, 41 (1): 59-65.

[16] Leclercy L. Calibration of flow-density relationships on urban streets [J]. Transportation Research Record: Journal of the Transportation Research Board, 2005, 1934 (1): 226-234. 